\title{
A Matter of Life and Death
}

\author{
Douglas R. Green \\ St. Jude Children's Research Hospital, Memphis, Tennessee 38105, USA \\ Correspondence: douglas.green@stjude.org
}

\section{CELL DEATH IS ESSENTIAL}

Every second, something on the order of 1 million cells die in our bodies. ${ }^{1}$ This is a good thing, because cell death is central to efficient homeostasis and adaptation to a changing environment. When, for some reason, it does not occur, the consequences can be catastrophic, manifesting as cancer, autoimmunity, or other maladies. Alternatively, if cell death occurs at the wrong time and place, this can also produce untoward effects, such as stroke, degeneration, heart attack, and many other injuries; thus, cell death is a major component of disease.

Cells die for a variety of reasons. For example, they can become physically or chemically stressed to the point that they cannot maintain their integrity. More often, however, such stresses engage an active cell death process before the stress becomes overwhelming; that is, the cell essentially commits suicide. Most, but not all, cell suicide occurs by a process we call apoptosis.
Another stress that triggers cell death is infection of the cell by an organism that would use the cell for its own parasitic ends (i.e., to make additional parasites). Such cell death can protect the body from further infection, unless the parasite can prevent this protective death until it has reproduced. In organisms with immune systems, lymphocytes and phagocytic cells can detect an infected cell and instruct the cell to die. This cell death can be apoptosis or another type of active cell death.

But it is not only in response to stress that cells die. In the course of normal development, cells can serve functions that become superfluous, and developmental cues (in the form of specific molecules) can signal such a cell to die. Technically, the death of a cell at a prescribed time in development is called programmed cell death, although this term is often applied to apoptosis in general, probably incorrectly. ${ }^{2}$ Another situation in which cell death occurs is when a cell acquires a mutation that allows it to lose its social inhibitions and prolif-

\footnotetext{
${ }^{1}$ We do not know the exact number, but if we consider only one type of cell, the neutrophil, it is estimated that up to 1 million of these cells die per second. The turnover of mucosal epithelium (in the lung and gut) is also very high. One million per second is probably an underestimate, but it is in the ballpark.

${ }^{2}$ The idea that apoptosis is "programmed" comes from early suggestions that activation of gene transcription is required for this form of cell death (which is sometimes, but not always, true, as discussed in other reviews in this subject collection). However, a distinction between programmed cell death and apoptosis is worth making because not all apoptosis is "programmed" by genetic events, and not all developmentally programmed cell death occurs by apoptosis. But this may be a losing proposition because a great many people use the terms interchangeably. In this book, we avoid the use of "programmed cell death" unless we are discussing specific developmental events. From the recent volume Cell Death: Apoptosis and Other Means to an End by Douglas R. Green

Additional Perspectives on Cell Death available at www.cshperspectives.org

Copyright (C) 2022 Cold Spring Harbor Laboratory Press; all rights reserved; doi: 101101/cshperspect.a041004 Cite this article as Cold Spring Harb Perspect Biol 2022;14:a041004
} 
D.R. Green

erate as a cancer. Tumor-suppressor mechanisms that are built into our cells instruct them to undergo suicide rather than imperil the body at large.

\section{TYPES OF CELL DEATH}

There are three major types of cell death ${ }^{3}$ : apoptosis, autophagic cell death, and necrosis. Apoptosis (sometimes called "type I cell death") means, literally, a "falling off." The term was coined to invoke leaves falling from a tree, because cells that die by apoptosis in tissues usually do so in an apparently random manner.

When cells die by apoptosis, the contents of the dying cell remain contained in membranes. The plasma membrane contorts into "blebs," and often the cell fragments into smaller membrane-bound "apoptotic bodies" (this term is also applied to apoptotic cell "corpses" that do not break up). The nucleus undergoes characteristic changes, including condensation, and it, too, often breaks up. Another characteristic feature of apoptosis is the cleavage of DNA, usually into pieces that are multiples of nucleosomes that appear as a "ladder" on agarose gels. Other organelles do not undergo dramatic morphological changes. The dying cell shrinks, and, if it is adherent, it detaches from surrounding cells. Some of the morphological changes associated with apoptosis are shown in Figure 1.

Apoptotic bodies are rapidly removed by other healthy cells through phagocytosis ("eating by cells"). In a relatively short time, nothing obvious remains to indicate that a cell had ever been there. Generally, apoptosis is not associated with a subsequent inflammatory response (but it can be in some settings).

\footnotetext{
${ }^{3}$ This is based on recommendations made by the Nomenclature Committee on Cell Death.

${ }^{4}$ Although it is often related that the first use of the term comes from Homer, a careful perusal of that esteemed author's works by Mauro Degli-Esposti failed to turn up this word. Instead, the first use of the term appears to be in the works of Hippocrates, who used it in a medical context 2400 years ago, referring to the "falling off" of bones in gangrene. It was used as well some 550 years later by the celebrated physician Galen and extended to the "falling off" of scabs.
}
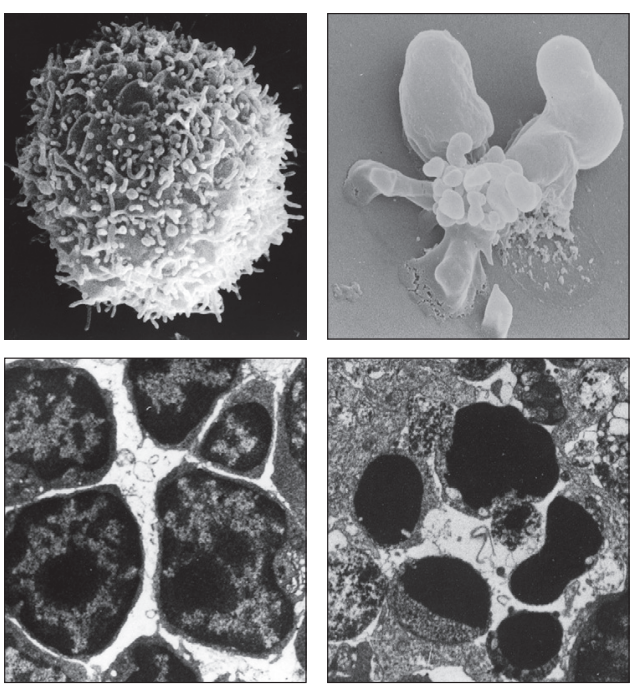

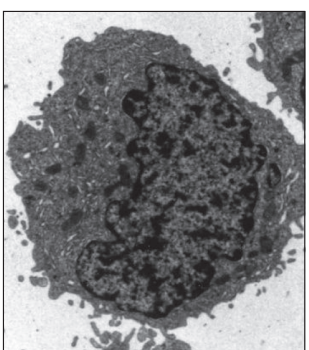

Living cell

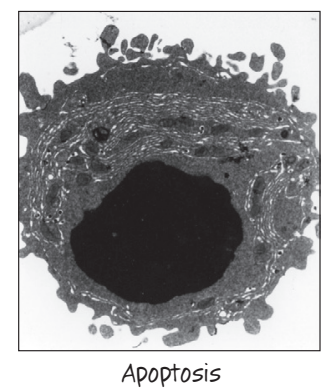

Figure 1. Apoptosis snapshots. (Images provided by Douglas R. Green.)

Autophagic cell death (type II cell death) differs from apoptosis and is less well understood. It involves a cellular mechanism called autophagy (literally, "self-eating") that is normally involved in sustaining cell metabolism under conditions of nutrient deprivation. Whether autophagy kills the cell by autophagic cell death is controversial, and most, but not all, studies suggest that autophagy accompanies such cell death in a last-ditch effort to keep the compromised cell alive. Often, this form of cell death occurs when, for some reason, apoptosis is blocked. Autophagic cell death is characterized by the appearance of vacuoles in the cell and does not include extensive condensation of the nucleus (Fig. 2).

The third major type of cell death-necrosis (also known as type III cell death) - is a messy 

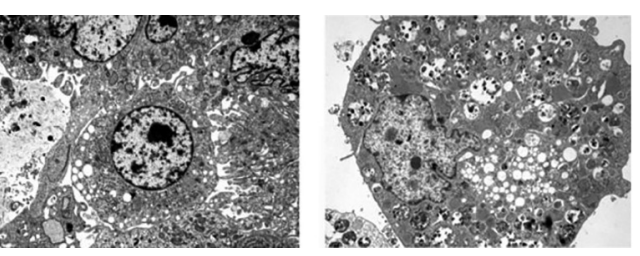

Figure 2. Autophagic cell death. (Left) The cell in the center is undergoing autophagic cell death. (Right) Another autophagic cell death. (Reprinted from Maclean et al. 2008. (C2008 with permission from the American Society for Clinical Investigation.)

sort of death in which the cell swells, bursts, and decomposes. It can occur when the plasma membrane is ruptured or when energy levels drop so quickly that the cells cannot sustain themselves (Fig. 3). However, there are forms of necrosis that are regulated by molecular processes; in other words, cells contain additional suicide pathways that result in necrosis rather than apoptosis.

There is another form of cell death worth mentioning, but it is very specialized. When
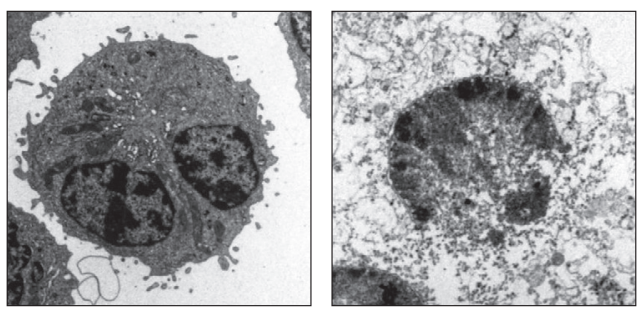

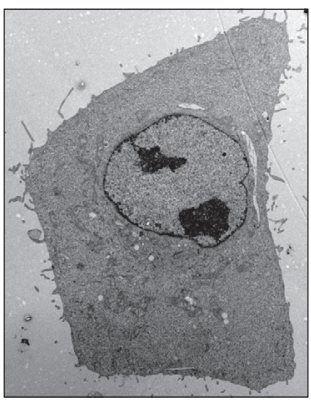

Living cells

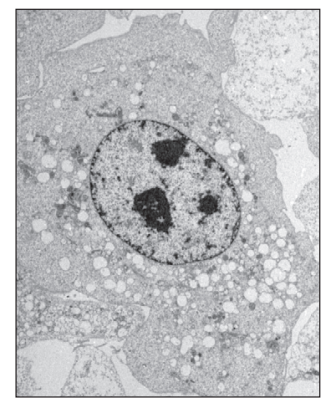

Necrosis
Figure 3. Necrosis. (Images courtesy of Dr. Nigel Waterhouse, Mater Medical Research Institute, Brisbane, Australia.) skin is created, epidermal keratinocytes die by a process called cornification; the nucleus is degraded and the proteins of the cell are extensively cross-linked to make a hard, dead material that has a very important barrier role in our bodies. This form of cell death is distinct from those we consider in this book, and, because it is a very specialized process, we do not discuss it further.

There are many other types of cell death that have been described, with terms such as pyroptosis, necroptosis, ferroptosis, entosis, aponecrosis, paraptosis, mitotic catastrophe, etc. In general, these do not qualify as unique classes of cell death, and thus we generally include them under the three major types of cell death outlined above.

\section{ACTIVE CELL DEATH: SUICIDE VERSUS SABOTAGE}

Another way we can think about cell death is whether it is active or passive. In passive cell death, the cell is damaged to the point that it simply gives up the ghost, and the cell does not participate in the actual death event. Alternatively, in active cell death, cellular processes have a role in the coup de grâce.

We can further parse active cell death into mechanisms that resemble suicide, in which the cell kills itself (this happens in all three types of death discussed above), or sabotage, in which the disruption of an active cellular process (not otherwise involved in cell death) kills the cell. Removing railway ties does not "kill" a train on the track unless that train is actively moving. ${ }^{5}$

The distinction might be useful in thinking about many different forms of cell death. It is tempting to think of cellular suicide as something that evolved as a means to engage cell death in response to a signal, whereas sabotage lacks such selective value. For example, it is possible (but not proven) that disruption of some

\footnotetext{
${ }^{5}$ This analogy relates to the origin of "sabotage," from "sabot," French for "wooden railway tie," and arguably the root of the term. Subsequently, it was related to sabotage of looms by Dutch weavers in the 15th century who would throw wooden shoes (also "sabot") to break the cogs, but the term did not appear until much later, when railway "sabotage" occurred.
} 
D.R. Green

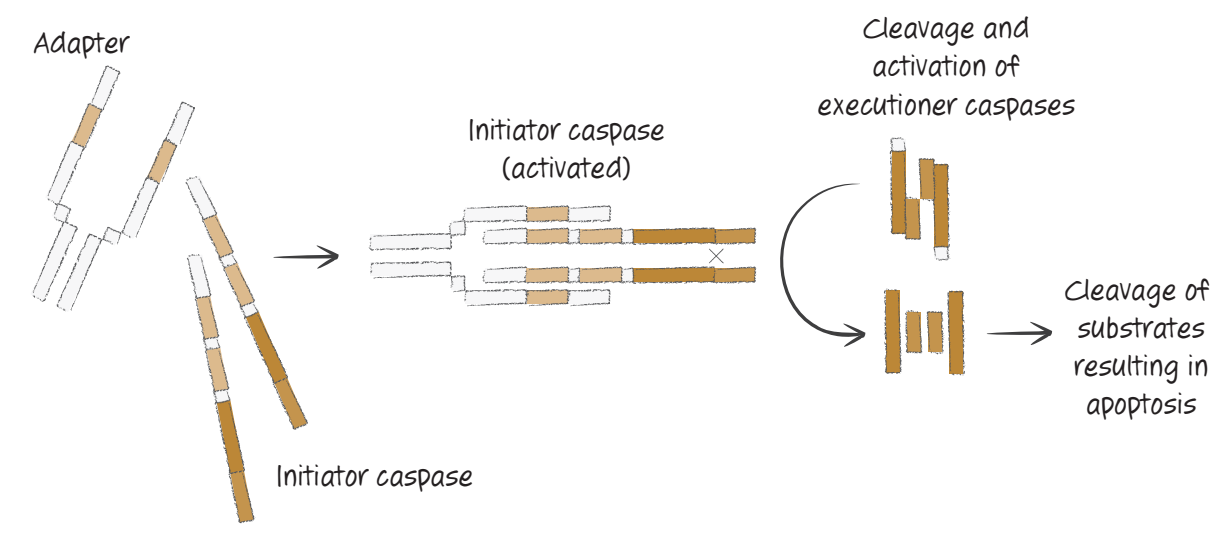

Figure 4. General caspase activation pathways.

parts of the cell cycle, or of normal metabolic processes, can kill cells in a manner that depends on the disrupted process being active, but such an active cell death by sabotage would not have been selected by evolution for that purpose. However, ultimately this is speculation, and the lines between these distinctions might blur.

\section{APOPTOSIS FROM THE "BOTTOM UP"}

Probably the best example of active, cellular suicide is apoptosis. The process of apoptosis is perhaps most easily understood by viewing it in reverse, beginning at the extreme end of a cell's life and tracking back to the healthy cell and what caused it to die. The "packaging" of the dying cell and the signals it sends to other cells to clear it away are brought about by the cleavage of many hundreds of different proteins in the cell. The proteases (protein-cutting enzymes) responsible for this cleavage are called caspases, and, in particular, they are referred to as a subset called "executioner caspases."

Executioner caspases are already present in healthy cells, but in an inactive form. They become activated when they are themselves cut by other proteases, called "initiator caspases." These, too, are present in healthy cells, but their activation mechanism is different. Initiator caspases are inactive until two identical chains are brought together by adapter proteins to produce an active enzyme ("adapter" is a general term used to describe proteins that bind to and bring together other proteins; we use the term here to refer to those that perform this function for the initiator caspase chains).

Different adapter proteins are engaged by different apoptotic stimuli and define distinct apoptotic pathways (Fig. 4). When adapters promote the activation of initiator caspases, the latter cut and thereby activate the executioner caspases. The executioner caspases then cleave hundreds of substrates, and the cell undergoes cell death and the morphological changes associated with apoptosis. The different pathways converge on the same set of executioner caspases, which is why our bottom-up view is warranted and is the way in which we unravel these pathways in other reviews in this subject collection.

\section{ROADS TO RUIN: THE PATHWAYS OF APOPTOSIS}

Most apoptosis in vertebrates (at least) occurs by what is termed the "mitochondrial pathway." In this pathway, conditions that promote cell death engage a set of related proteins, called the BCL-2 family (Fig. 5), that control the integrity of the outer membranes of mitochondria in the cell. Proapoptotic BCL-2 effectors disrupt outer mitochondrial membranes, whereas antiapoptotic BCL-2 proteins prevent this disruption and thereby prevent apoptosis. A third set of BCL-2 proteins regulates the other two types.

If mitochondrial outer membrane permeabilization (MOMP) occurs, soluble proteins of 
A Matter of Life and Death

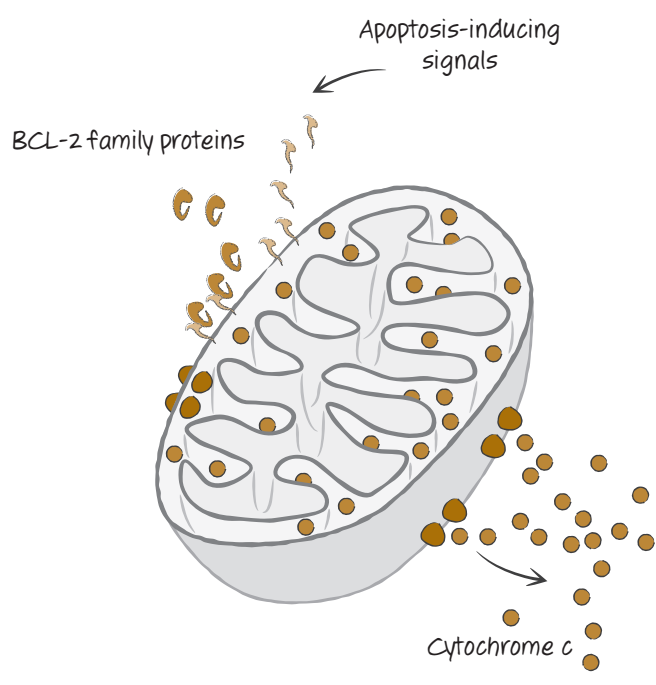

Figure 5. Simplified scheme of the first part of the mitochondrial pathway. BCL-2 proteins control mitochondrial outer membrane permeabilization (MOMP), releasing proteins that include cytochrome $c$. the intermembrane space (between the outer and inner mitochondrial membranes) diffuse into the cytosol. These proteins include cytochrome $c$, which also has a central role in mitochondrial physiology.

When cytochrome $c$ reaches the cytosol, it interacts with an adapter protein that is present there, causing the adapter to cluster (oligomerize) and bind to monomers of one of the initiator caspases. The initiator caspase is thus activated (Fig. 6) and, in turn, cleaves and thereby activates executioner caspases that themselves go on to cleave their substrates. Apoptosis ensues.

A second pathway for caspase activation and apoptosis involves specialized receptors on the cell surface, called death receptors. When the ligands for these receptors bind, the intracellular region of these receptors engages a specific adapter molecule (distinct from that of the mitochondrial pathway). This, in turn, binds to

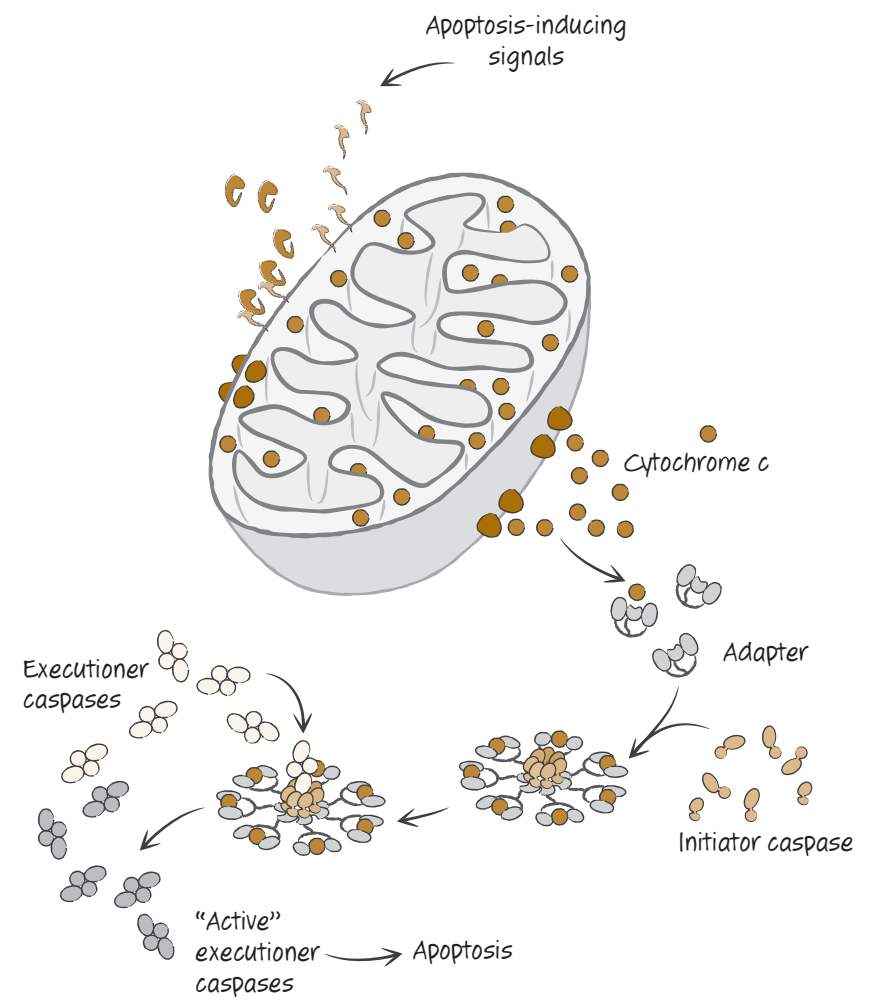

Figure 6. Simplified scheme of the mitochondrial pathway of apoptosis. 
D.R. Green

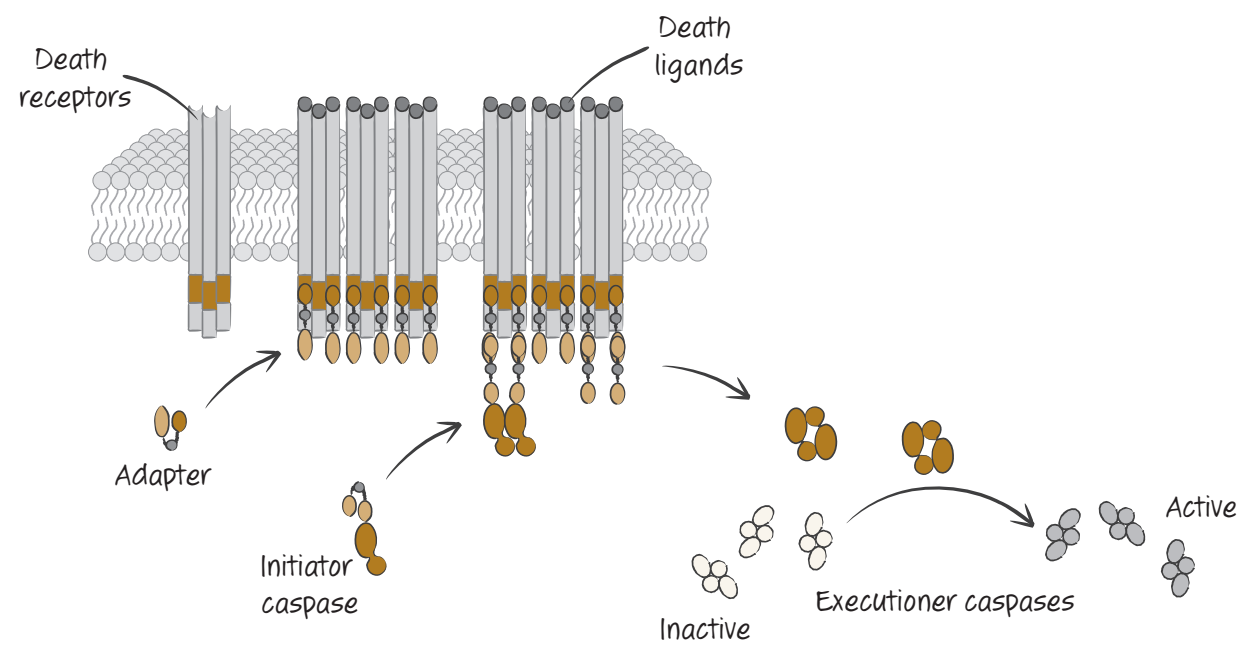

Figure 7. Simplified scheme of the death-receptor pathway of apoptosis, in which death receptors on the plasma membrane bind their ligands and, through several intermediaries, ultimately activate executioner caspases.

monomers of an initiator caspase (different from that of the mitochondrial pathway), activating it. The initiator caspase cleaves and thereby activates executioner caspases (the same as above), and apoptosis proceeds (Fig. 7).

As mentioned earlier, another type of signal that can induce cell death is infection of a cell. Our cells have sensors that detect such infection, and some of these also act to engage adapters (distinct from those of other pathways) for a caspase related to initiator caspases. Unlike the initiator caspases, however, this caspase (and another discussed in Green 2022) can also cleave a specific substrate that causes, not apoptosis, but a form of necrosis called pyroptosis. It also processes and allows the secretion of mediators that engage host defenses to fight the infection (Fig. 8).

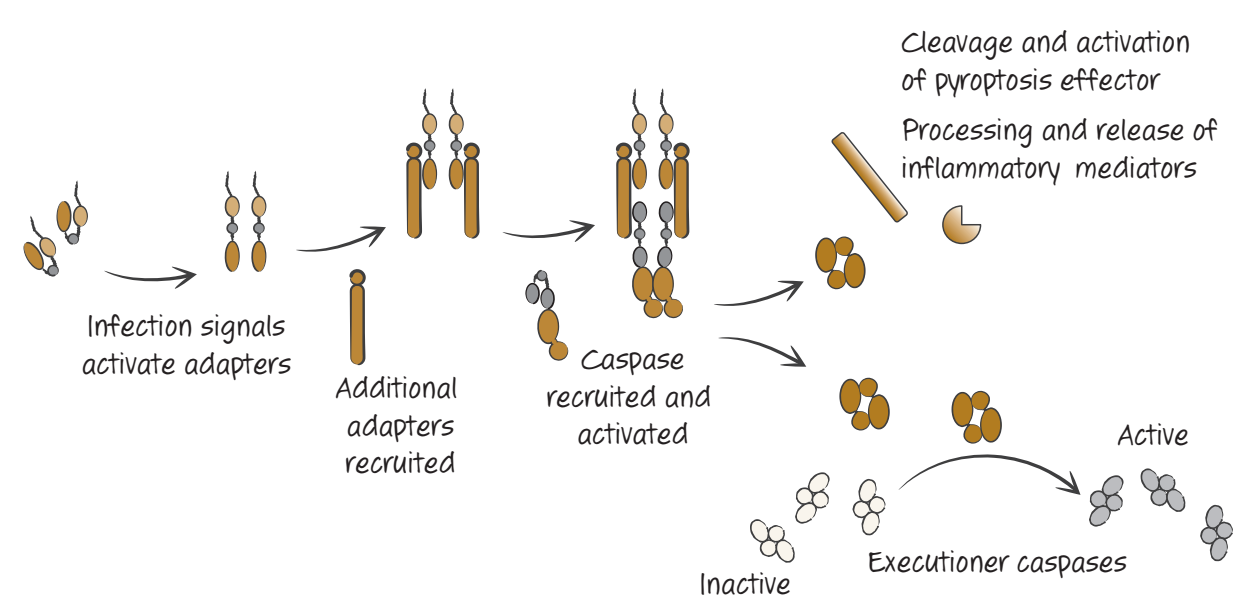

Figure 8. Simplified inflammasome, showing events leading to the twin events of activation of executioner caspases and the secretion of inflammatory mediators. 


\section{A GENERAL PROBLEM IN CELL DEATH RESEARCH}

What death is might seem obvious. But, in practice, cell death turns out to be a bit tricky to define in a general way. If a cell loses its plasma membrane integrity, it is certainly dead, but, as we mentioned above, apoptotic cells are often engulfed before this event, and a cell that has been eaten and is being digested is dead, too. However, if we wait until that event to register this as a "cell death," we face a kinetic problem, because some cells will be long gone before others engage the pathway, and any count of dying cells could be an underestimate. Therefore, we might prefer to call a cell "dead" if it has activated caspases and is undergoing apoptosis. However, here we have additional problems because it often happens that cells that initiate apoptosis might not die (if, e.g., the extent of caspase activation is low) or, if caspases are experimentally blocked, death ensues nevertheless (although without the features of apoptosis). We might count a cell as "dead" if it loses the ability to proliferate or perform its functions. However, cells that lose the ability to proliferate can persist for years (indeed, this is very important for many of our cells), and functions can change. So, when is a cell "dead"?

In practice, the definition of "death" is often tied to the cells and systems under study. Therefore, surrogate markers of cell death are often used, and it is assumed that these represent cellular demise. Although extensive progress has been made using this approach, mistakes abound. Undoubtedly, possible mistakes in this book might themselves be the result of the erroneous interpretation of such surrogates as "death," and it is advisable, therefore, to keep this issue in mind.

The details of these apoptosis pathways are discussed in much more detail in the rest of this book, but the basics of the pathways are as described here. As we will see, apoptosis in other animals also follows these general schemes of adapter-caspase interactions.

\section{THE “TOP” OF THE PATHWAY: WHAT INDUCES APOPTOSIS?}

The pathways we have briefly considered above are triggered by distinct types of signals. Most events that stress a cell engage the mitochondrial pathway of apoptosis. These include loss of growth factors, agents that damage DNA, disruption of the cytoskeleton, extensive protein aggregation, and other forms of cell stress. This pathway is also often triggered by developmental signals and by tumor-suppression mechanisms. The death-receptor pathway is engaged by the specific ligands that bind to the receptors. The inflammasome pathway is induced by signals resulting from infection, although some inert substances can act in the same way if taken up into the cell. Therefore, apoptosis is engaged by a wide range of conditions, and there can be extensive overlap with regard to which pathway can be trig- gered by a specific agent, depending on the type of cell. As we will also see, these pathways can also "cross talk," in that the death-receptor pathway or inflammasome pathway can engage the mitochondrial pathway as well.

\section{DYING CELLS ARE SIGNALS}

When a cell dies, other cells in the body rapidly remove the corpse. ${ }^{6}$ Cells that undergo apoptosis produce signals, as a consequence of caspase activation, that attract cells capable of eating the dying cell and also other signals that cause removal of the apoptotic cell before the plasma membrane is disrupted. Necrotic cells, in contrast, release intracellular molecules that also lead to their clearance, but in a way that promotes inflammation of the tissue. Depending on how the cell died, our immune system can

\footnotetext{
${ }^{6}$ We seem to be violating our "bottom-up" view by discussing the consequences of cell death (admittedly, the "bottom") only after discussing the "top" of the pathway. But cells that die by any mechanism, not only apoptosis, can have an impact on physiology.
} 
D.R. Green

therefore be engaged to respond to whatever might have caused the cell death. Alternatively, it can be instructed not to respond.

It is evident that the ways in which dying cells affect the body are complex, and the consequences of cell death are varied-and when a cell is gone, it might not be forgotten.

\section{REFERENCE}

${ }^{*}$ Reference is also in this collection.

* Green DR. 2022. Inflammasomes and other caspase-activation platforms. Cold Spring Harb Perspect Biol doi:10 $.1101 /$ cshperspect.a041061

\section{ADDITIONAL READING}

\section{Cell Death Nomenclature}

Andre N. 2003. Hippocrates of Cos and apoptosis. Lancet 361: 1306.

A note on an early use of "apoptosis" in medicine.

Degli-Esposti M. 1998. Apoptosis: who was first? Cell Death Differ 5: 719.

A hunt for the origins of the word "apoptosis."

Galluzzi L, Bravo-San Pedro JM, Vitale I, Aaronson SA, Abrams JM, Adam D, Alnemri ES, Altucci L, Andrews D, Annicchiarico-Petruzelli M, et al. 2015. Essential versus accessory aspects of cell death: recommendations of the NCCD 2015. Cell Death Differ 22: 58-73.

More than simply nomenclature, this review outlines the different types of cell death and problems with their classification.

Green DR, Victor B. 2012. The pantheon of the fallen: why are there so many forms of cell death? Trends Cell Biol 22: $555-556$.

A potentially useful way to think about different types of cell death.

\section{History of Apoptosis}

Diamantis A, Magiorkinis E, Sakorafas GH, Androutsos G. 2008. A brief history of apoptosis: from ancient to modern times. Onkologie 31: 702-706.

Not essential reading, but an interesting overview of the study of apoptosis.

Horvitz HR. 2003. Nobel lecture. Worms, life and death. Biosci Rep 23: 239-303.

The modern study of apoptosis was ignited by the identification of genes that control cell death during development in the nematode Caenorhabditis elegans. This is the Nobel lecture that describes those studies.

Kerr JF, Wyllie AH, Currie AR. 1972. Apoptosis: a basic biological phenomenon with wide-ranging implications in tissue kinetics. Br J Cancer 26: 239-257.

This is the original paper formally describing apoptosis.

Saunders JW Jr. 1966. Death in embryonic systems. Science 154: 604-612.

A classic early overview of the role of cell death in development.

Wyllie AH, Kerr JF, Currie AR. 1980. Cell death: the significance of apoptosis. Int Rev Cytol 68: 251-306.

An early review that details the state of knowledge on apoptosis before molecular mechanisms became understood.

Lockshin RA. 2016. Programmed cell death 50 (and beyond). Cell Death Differ 23: 10-17.

History and perspectives on cell death from the author who coined the term "programmed cell death."

Green DR. 2016. The cell's dilemma, or the story of cell death: an entertainment in three acts. FEBS $J$ 283: 2568-2576.

Just for fun, a play about the study of cell death; where we've been, where we are, and where it might be going.

\section{FIGURE CREDIT}

Maclean KH, Dorsey FC, Cleveland JL, Kastan MB. 2008. Targeting lysosomal degradation induces p53-dependent cell death and prevents cancer in mouse models of lymphomagenesis. J Clin Invest 118: 79-88. doi:10.1172/ JCI33700 


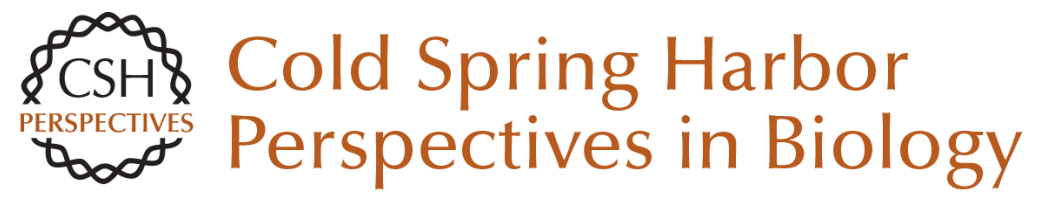

\section{A Matter of Life and Death}

Douglas R. Green

Cold Spring Harb Perspect Biol 2022; doi: 10.1101/cshperspect.a041004

\section{Subject Collection Cell Death}

\section{The Future of Death} Douglas R. Green

Caspase Activation and Inhibition Douglas R. Green

The Mitochondrial Pathway of Apoptosis Part II: The BCL-2 Protein Family Douglas R. Green

\section{Cell Death in Development} Douglas R. Green

The Death Receptor Pathway of Apoptosis Douglas R. Green

\author{
Cell Death and Cancer \\ Douglas R. Green \\ Inflammasomes and Other Caspase-Activation \\ Platforms \\ Douglas R. Green
}

The Mitochondrial Pathway of Apoptosis: Part I:

MOMP and Beyond Douglas R. Green

Caspases and Their Substrates

Douglas R. Green

A Matter of Life and Death

Douglas R. Green

For additional articles in this collection, see http://cshperspectives.cshlp.org/cgi/collection/

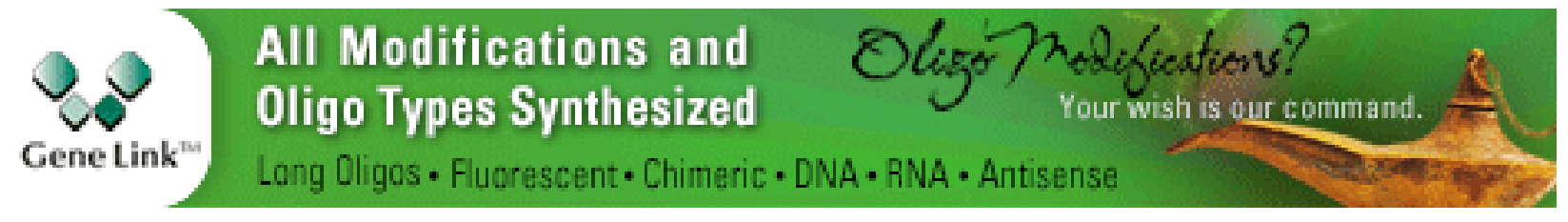

Copyright @ 2022 Cold Spring Harbor Laboratory Press; all rights reserved 